\title{
ACTIVISMO MEDIOAMBIENTAL MULTIMODAL EN EL TRIÁNGULO NORTE DE CENTROAMÉRICA: MEDIOS DIGITALES, PATRIMONIO BIOCULTURAL Y DE-COLONIALIDAD
}

\author{
MULTIMODAL ENVIRONMENTAL ACTIVISM \\ IN THE NORTHERN TRIANGLE OF CENTRAL \\ AMERICA: DIGITAL MEDIA, BIO-CULTURAL \\ HERITAGE, AND DE-COLONIALITY
}

\author{
LaURA Barbas RHOden \\ Wofford College
}

\begin{abstract}
RESUMEN
El presente trabajo se plantea en función de la consideración de una nueva forma de organización, formación y comunicación ciudadana que se ha movilizado en defensa del patrimonio biocultural en el Triángulo norte de América Central: la creación y el uso de espacios digitales como parte de un activismo medioambiental multimodal. En manos de diversas organizaciones y redes de grupos con intereses comunes, el espacio digital vuelve a ser un espacio generativo y disruptivo: generativo en que es propicio para la creación de identidades, discursos y archivos elaborados desde múltiples posiciones y disruptivo en que es apto para la difusión de epistemologías y éticas alternativas a las hegemónicas.
\end{abstract}

Palabras clave: medios digitales; Centroamérica; medio ambiente; ecocrítica; sociedad red.

\begin{abstract}
This paper considers a new form of organization, education, and communication that has been mobilized in defense of biocultural heritage in the northern triangle of Central America: the creation and use of digital spaces as part of a multimodal environmental activism. In the hands of various organizations and networks of groups with common interests, the digital space is at once a generative and disruptive space: generative in that it is conducive to the creation of identities, discourses, and archives created by multiple actors, in different positions, and disruptive in that it serves as a means of dissemination of alternative epistemologies and ethics.
\end{abstract}

Keywords: digital media; Central America; environment; ecocriticism; network society 
24.000 galones de agua por hora: es el consumo de agua de la mina metálica promedio, equivalente a lo que una familia salvadoreña promedio utiliza durante 20 años (Achtenberg 3). El número de asesinatos en 2012 en Guatemala (35/100.000 habitantes), Honduras (91/100.000 habitantes) y El Salvador (41/100.000 habitantes) excede el nivel epidémico según la Organización Mundial de Salud (Doggett 72; World Bank); y ha habido más de 100 asesinatos de activistas medioambientales en Honduras entre 2010-2014 (Global Witness). Son las realidades político-sociales y medioambientales que enfrentan las comunidades del istmo a diario en un entorno moldeado por modelos extractivistas, políticas neoliberales y una persistente inequidad a nivel nacional. El presente trabajo se plantea en función de la consideración de una nueva forma de organización, formación y comunicación ciudadana que se ha movilizado en defensa del patrimonio biocultural en el Triángulo Norte de América Central: la creación y el uso de espacios digitales como parte de un activismo medioambiental multimodal. En manos de diversas organizaciones y redes de grupos con intereses comunes, el espacio digital vuelve a ser un espacio generativo y disruptivo: generativo por ser propicio para la creación de identidades, discursos y archivos elaborados desde múltiples posiciones y disruptivo por ser apto para la difusión de epistemologías y éticas alternativas a las hegemónicas que rigen los acuerdos transnacionales de comercio y que han dado forma a las estructuras legales, políticas y financieras que han acelerado la depredación tanto de la tierra como de las poblaciones humanas en la era neoliberal.

En las primeras décadas del Siglo XXI, en varios sitios alrededor del mundo, los recursos digitales han sido parte de una práctica multimodal mediante la cual redes de personas se movilizan alrededor de temas de importancia para ellos, ya sea por su ubicación (territorio compartido) y/o su afinidad (interés o identidad común). Este tema se ha estudiado en el contexto de la Primavera Arabe, el movimiento 15-M en España y también el movimiento Occupy Wall Street, entre otros (Bennett y Segerberg; Castells Networks). Si bien comparte mucho con otros movimientos, el fenó- 
meno digital emergente en Centroamérica nos obliga a considerar nuevos interrogantes: ¿̇cuáles son las características definitorias de la movilización multimodal $y$, en particular, su trabajo mediado digitalmente, en una zona marcada por altos índices de violencia, impunidad y corrupción? ¿̇Hasta qué punto y cómo se interrelacionan diferentes actores -desde antiguas redes de solidaridad transnacional con raíces en la época revolucionaria a organizaciones comunitarias locales, en redes digitales que se activan en relación con temas ambientales? ¿ Con qué retórica (tanto discursos como retórica visual) articulan posiciones y en relación con qué referentes del mundo biofísico plantean sus argumentos los que intervienen en defensa de la herencia eco-biológica y cultural de sus territorios ante cambios dramáticos y devastadores en la presente época?

\section{Aproximación académica a la movilización medioambiental centroa- MERICANA}

En el presente ensayo traslado los trabajo de Manuel Castells sobre comunicación en "la sociedad red" (2006; 2009; 2012); el de Lance Bennett y Alexandra Segerberg sobre la acción conectada (2013); y el de Rita Raley sobre los medios tácticos (2009), al campo específico del activismo medioambiental en la Centroamérica contemporánea y, en particular, a los tres países con los más altos índices de violencia, inequidad e impunidad en el istmo.

Dentro de las movilizaciones a favor de la vida (humana, ecológica, comunitaria) en el Triángulo Norte, el uso de los espacios digitales como herramienta de lucha ha abierto un lugar de contrapoder para grupos que luchan a favor de derechos a la tierra, el agua y una voz pública brindándoles un nuevo recurso para superar barreras institucionales y participar en debates de los que antaño habían sido excluidos. A pesar de incorporar análisis de documentos, lo que propongo antes que nada en el presente ensayo es un acercamiento preliminar al activismo medioambiental multimodal centroamericano que señale sus características generales y sitúe sus intervenciones en el panorama de actos de descolonización y auto-determinación en el nuevo orden social de la sociedad red. Para llevar a cabo mi investigación, he examinado material digital de 18 grupos cuyos comunicados indican agendas medioambientalistas en el Triángulo Norte; he leído textos secundarios sobre las zonas y los temas que han impulsado la movilización de grupos y redes de acción; y he utilizado herramientas digitales de análisis digital de textos (conteo de palabras, asociación/relación entre palabras), redes de temas (relación en el Internet entre grupos de interés común). 
Frente a las preguntas ¿̇por qué amerita una indagación académica este fenómeno de la movilización multimodal en defensa del patrimonio biculturale y ¿̇por medio de qué acercamiento teórico y disciplinario hacerlo? surgen varias aproximaciones posibles y cabe destacar que el hecho de responder críticamente a nuevos fenómenos culturales latinoamericanos de tendencia medioambiental requiere que se amplíe la definición de producción cultural así como la de "ecológico" o "medioambiental"; que se considere la multiplicidad de formas en que los individuos y colectividades responden a los cambios que perciben en el mundo; y que se reconozca de que estas intervenciones pueden tomar la forma de actos individuales, colectivos y/o conectados (Bennett y Segerberg 13). En cuanto a abogar por una reorientación del campo de estudio denominado "verde" para que sea más inclusivo de la heterogeneidad cultural del mundo globalizado, coincido con Gisela Heffes quien en relación con la ecocrítica ha aseverado que "una nueva episteme crítica debe incluir, además de una ética más madura y reflexiva, las intersecciones entre la metrópolis y el interior, como así también la conjugación entre preocupaciones antropocéntricas, biocéntricas y ecocéntricas" (71). Este fenómeno cultural - del uso de nuevos medios digitales como componente importante de la respuesta ciudadana ante la crisis medioambiental en territorios centroamericanos - es precisamente una de las prácticas que insisten en ampliar los parámetros del campo de investigación de las humanidades digitales, estudios de nuevos medios y estudios culturales en la academia, para que se considere a fondo su uso en zonas marginadas, ya sea en el interior de cualquier país (en contraste con la capital-metrópoli) o desde la periferia del sistema-mundo en términos de Immanuel Wallerstein (1974).

La praxis digital centroamericana heterogénea, iterativa, formulada con base en diversas alianzas laterales (entre individuos empleados en diferentes sectores, desde el sector comunitario y sin fines de lucro al artístico y periodístico) y de dinámica constante entre interior-metrópolis-exterior, me ha obligado a armar un aparato de análisis sintetizado de estudios en múltiples campos y disciplinas. De las ciencias, han iluminado mi trabajo recientes estudios multidimensionales sobre el mundo biofísico centroamericano, el cual funciona como referente para los grupos y alianzas. De la antropología, sociología y geografía han beneficiado mi trabajo investigaciones sobre la interacción a diario de diversas poblaciones con su entorno biofísico así como postulaciones como las de Castells y Bennett y Segerberg sobre cómo se transmiten mensajes y se movilizan los individuos en el mundo red. Mi previa investigación en la historia económica, los estudios culturales latinoamericanos y las teorizaciones sobre temas de relevancia medioambiental en la producción cultural de los Estados Unidos, 
Europa y América Latina, para mi libro Ecological Imaginations in Latin American Fiction (201 1), dan cuenta de mi comprensión sobre la consciencia medioambiental dentro de una compleja dinámica nacional, regional y transnacional. En cuanto al estudio de medios digitales como tácticas desestabilizadoras, me ha sido imprescindible el trabajo de Rita Raley, ya que las intervenciones digitales de los grupos y alianzas que estudio en el presente ensayo, son prácticas estéticas y culturales que buscan interrumpir el status quo de consumismo basado en la explotación de recursos naturales y humanos.

A diferencia de los fenómenos digitales que estudia Raley, algunos de los cuales precisan de tecnología costosa y acceso a información no disponible en Centroamérica, hasta el momento las formas de comunicación digital de los movimientos que estudio son de "bajas barreras de entrada", o sea tecnología de bajo costo, fácil acceso y que no requiere entrenamiento especializado (vídeos grabados en teléfonos móviles; blogs, sitios web, páginas de Facebook, cuentas de Twitter y YouTube). Con la emergencia de más tecnologías e información de libre acceso (como el mapeo utilizando datos generados por individuos o tomados de registros públicos en plataformas como Open Street Maps), así como la evolución del emergente campo de humanidades digitales en América Latina, el potencial generativo y disruptivo de movimientos ya digitalizados seguirá cobrando importancia.

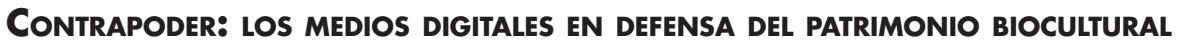

Como bien nota Castells, el contexto histórico de las primeras décadas del Siglo XXI se caracteriza por dos fenómenos interrelacionados: los procesos de globalización y el auge de la sociedad red, cada uno de los cuales depende de redes de comunicación que procesan conocimiento y pensamiento para estimular la confianza entre entidades y facilitar el intercambio de información, bienes y capital (Communication 16). La confianza, según sostiene Castells, es el substrato del poder (Communication 16) y sin ella, no es posible llevar a cabo las operaciones diarias del poder sin recurrir a la violencia, sobre la cual generalmente tiene un monopolio, en términos de legitimidad, el Estado. A la observación de Castells, añadiría un adicional en relación al presente tema: que la relación entre (1) la confianza entre individuos y/o entidades (entre ciudadanos y gobiernos; entre un gobierno y otro; entre consumidores, productores y distribuidores) y (2) la legitimación de actos, en particular actos tomados por poderosas entidades transnacionales, depende de narrativas fundacionales heredadas tanto de épocas anteriores (la de la supremacía cultural anglo-europea, por ejemplo) como de las nuevas (las tecno-utópicas, las de la aldea global) que 
producen la conformidad, pasividad y complicidad de numerosas de las masas. Y aquí, en esta dinámica comunicativa, se inserta el fenómeno del contrapoder de parte de organizaciones y alianzas activistas que operan en espacios digitales con actos de comunicación que recurren a diferentes tropos y narrativas (algunos también heredados de épocas anteriores, como el tropo de la heroica resistencia indígena a la conquista europea) y que actúan para persuadir a sus receptores de cuestionar las premisas de los mensajes que se transmiten en el entorno cultural en que viven.

¿Por qué vuelve a ser tan disruptivo este conflicto narrativo que a lo mejor siempre ha existido en las sociedades humanas? Una mirada a la acción multimodal centroamericana revela que es disruptiva en parte por la democratización del poder narrativo que permiten los medios digitales y en parte, por motivos de escala; es posible crear narrativas, en formas convincentes (como testimonios en vídeo sobre actos de agresión) y difundirlas por redes pre-existentes y nuevas casi instantáneamente. Pongamos por caso el asesinato del 3 de marzo de 2016 de la líder indígena Berta Cáceres, ganadora del Premio Goldman en 2015, en Honduras; las primeras noticias de alcance internacional de su violenta muerte llegaron de parte de la Coordinación General de organización de la cual había sido co-fundadora, el Consejo Cívico de Organizaciones Populares e Indígenas de Honduras (COPINH), y horas después, era un "trending topic" en Twitter, entre los diez más difundidos a nivel mundial. El discurso oficial de autoridades locales, que aseguraba que se trataba de un intento de robo a la casa donde la víctima se alojaba con familiares en el pueblo La Esperanza (La Prensa), carecía de credibilidad desde el momento en que llegó al público internacional, ya que lo anticipaban centenares de tuits, actualizaciones en Facebook y mensajes de listas académicas y populares con enlaces a vídeos en los que Berta Cáceres denunciaba las amenazas a su vida.

Grupos activistas ubicados en territorios marginados en el sistema productivo global aprovechan los medios digitales, como lo ha hecho CO$\mathrm{PINH}$, por ejemplo, no sólo para desmentir discursos oficiales sino también para hacerse ver y escuchar, acto sumamente disruptivo en un sistema en el que son de otro modo considerados "irrelevantes" (Castells, Communication 26). Cobran importancia en el sistema global extractivo, como el sector minero resistido por muchas movilizaciones en el Triángulo Norte, al revelar a los ciudadanos-consumidores que dan legitimidad a procesos y actos de creación de valor financiero con sus compras e inversiones, aquello que les es invisible debido a una cadena de valores/suministros compleja y globalizada. Cada objeto físico que se compra tiene un locus de creación y llega al consumidor a través de una cadena de suministros 
de logística muchas veces compleja, así como cada creación de valor en el sistema financiero tiene un proceso - arraigado en la confianza y complicidad de millones de personas - aunque no vean tal proceso. Sin embargo, las redes de activismo anti-minero, por ejemplo, cortan estas distancias cuando difunden noticias de la violenta represión contra organizaciones comunitarias en Guatemala y organizan campañas para presionar al gobierno canadiense de quitarle fondos del plan estatal de pensiones de inversiones en compañías mineras como Tahoe Resources ("Mining Watch Calls on Canada Pension").

En una zona marcada por una grave inequidad, una larga historia de jerarquía social con base en el etnocentrismo colonial, un legado de gobiernos autoritarios y represión estatal, esta nueva herramienta de "contrapoder" (Castells, Communication 47-49), la de los espacios y las redes digitales, intenta ir contra tradicionales estructuras y medios de control y represión. Fortalece la organización ciudadana en forma horizontal y descentralizada a la vez que socava mecanismos de control: facilita el acceso a la información sobre el patrimonio cultural, peligros, abusos y amenazas, lo cual no permite el monopolio de los medios masivos sobre los mensajes que llegan al público; hace posible la creación y diseminación instantánea de información por parte de gente común y corriente, lo cual dificulta la represión, ya que crea un público más amplio de testigos y una red de participantes prevenidos; y sirve como archivo de testimonios, fotos, vídeos, mapas y acciones solidarias, lo cual subvierte el control de la producción y reproducción institucionalizadas de una cultura (nacional) legítima, legitimada y de acceso restringido en museos, archivos, libros escolares y cánones literarios.

El poder de las movilizaciones medioambientales como las de la Mesa frente a la Minería en El Salvador, el Consejo Cívico de Organizaciones Populares e Indígenas de Honduras (COPINH) en su lucha por la preservación del Río Gualcarque y el Comité de Unidad Campesina (CUC) de Guatemala en sus campañas anti-mineras, entre otros grupos y movilizaciones, viene en parte del uso de medios digitales como actividad integral de sus movilizaciones. Si bien el poder depende del control de comunicación, como asevera Castells, el contra-poder depende de la capacidad de romper e interrumpir tal control (Communication 3). El poder de estas intervenciones reside en la combinación de su capacidad disruptiva y generativa a escala local y transnacional. Para resumir, el uso de medios digitales en manos de grupos y redes de alianzas movilizados para fines medioambientales:

-elimina las distancias entre el lugar de extracción-producción y el lugar de consumo; 
- crea espacios compartidos que evitan el control efectuado por las jerarquías nacionales y los acuerdos de conveniencia entre élites nacionales y élites de la clase financiera multinacional;

- plantea la existencia, en forma retórica, de dos modos de consumo, el extractivista a servicio de sociedades adquisitivas ubicadas en "otro espacio", y una alternativa, un modelo de subsistencia al servicio de las necesidades humanas básicas como el acceso al agua, la comida y el hogar. Estos dos modos de consumo parten de diferentes modos de ser (ontologías), saber/conceptualizar (epistemologías) y experimentar el mundo.

A diferencia de las formas de producción cultural anteriores, el activismo digital dispone de nuevas formas de lo que Rita Raley llama "modes of reader-user engagement" [nuevos modos de compromiso lector-usario] (Raley 5) para desestabilizar la psicología del mercado. Las intervenciones digitales ambientalistas centroamericanas nacen de un rincón del planeta globalizado distinto del locus de creación de los medios tácticos que estudia Raley y, a pesar de compartir las mismas tendencias disruptivas y educativas, hacen algo muy propio de su lugar geopolítico: visibilizan actos de destrucción biocultural; sirven como repositorios de memorias de resistencia y contestación y encarnan otros modos de saber, ser y actuar en el mundo que por sus actos, vuelve a ser más pluriverbal.

\section{Activismo a favor del patrimonio biocultural en el Triángulo Norte}

Consideremos ahora de cerca expresiones del fenómeno del activismo multimodal centroamericano en defensa del patrimonio biocultural. (Ver Figura 1.) Antes que nada, cabe resaltar que el activismo medioambiental es (1) multimodal, (2) de temas múltiples e integrados y (3) que nace de un contexto sociohistórico y geopolítico particular. ¿̇En qué sentido es el movimiento multimodal? Lo identifico como movimiento multimodal porque los participantes utilizan diferentes modos de comunicación y acción, desde los tradicionales en términos organizativos (como la formación de grupos comunitarios, manifestaciones en la calle) hasta las nuevas, posibilitadas por la sociedad red (videos testimoniales difundidos en redes sociales, campañas de hashtag). Una movilización multimodal puede amplificar acciones tomadas en diferentes espacios (tanto los físicos como los virtuales). Por ejemplo, bloquear una carretera, acto común en las movilizaciones comunitarias en América Latina, es un acto de apropiación del espacio físico y simbólico de la infraestructura comercial del país; al difundirse por redes sociales en vídeos y fotos tomados por participantes en la acción, la protesta adquiere más testigos y puede motivar actos solidarios. La movilización multimodal como fenómeno cultural ante amenazas al patrimonio 
biocultural nace entonces de consideraciones prácticas y filosóficas por parte de organizadores tradicionales, como comités comunitarios, y de la postura ética de participantes que quieren fomentar intercambios inclusivos con diferentes actores con varios niveles de participación digital. Por eso se ve comúnmente en las redes que estudio aquí la práctica de ir casa por casa con información, de tener o formar colaboraciones con radioemisoras locales, de compartir mensajes por medio del teatro, así como comunicarse con investigadores en centros urbanos, universidades nacionales o en el exterior y otros colaboradores usando redes sociales y plataformas para la difusión de información.

Ahora, ¿̇por qué consiste este movimiento de temas múltiples e integrados? La experiencia vivida de los participantes revela un nexo con problemas interrelacionados que son difíciles de separar: marginación del interior del país vis-a-vis la metrópoli; orientación de la política económica nacional, durante siglos, hacia la exportación; dominio en la política nacional por parte de una élite (oligarquía) nacional, con débiles instituciones civiles como consecuencia; depredación de los recursos necesarios para el cultivo de comestibles para consumo nacional; marginación de pequeños productores. Nacen estos problemas de un contexto sociohistórico y geopolítico particular y el trabajo de los participantes (tanto organizaciones como individuos) en movimientos multimodales es el de impulsar avances en múltiples áreas, algo que se ha observado en otros movimientos como Occupy Wall Street que tuvieron lugar alrededor del tema del cambio climático (Bennett y Segerberg 6-7).

En la sección que sigue considero algunas redes de acción medioambiental multimodal en cada país del Triángulo norte. Al mirar de cerca un movimiento y/u organización/red de organizaciones, considero su retórica e iconografía en comunicación multimodal y para ofrecer una lectura de un producto de cada movimiento de alcance internacional: una muestra de retórica visual o un discurso, también menciono, pero no analizo, vídeos documentales. Después de señalar brevemente qué pasa en cada país, sigue una discusión extensa sobre las características generales y las implicaciones de las movilizaciones.

\section{El Salvador}

Con una población en 2000 de 6,3 millones de habitantes, El Salvador es el país más densamente poblado en el continente americano, con 315 personas por kilómetro cuadrado (Blackman, et.al. 28). Se estima que más del $20 \%$ de la población vive fuera del país (Benítez 1440). Menos del $10 \%$ de sus bosques naturales sobreviven y el país está último o penúltimo 
en el rango en las Américas para la desforestación (Blackman, et.al. 24). En términos de política ecológica ha habido proyectos de ley ambiental importantes a finales del Siglo XX, como en otros países centroamericanos: se aprobó en 1998 una ley medioambiental y en el mismo año también se creó el Ministerio de Medio Ambiente y Recursos Naturales, o MARN, que tiene la obligación de dar permiso para cualquier cambio en el uso de la tierra. Copias de varias leyes ambientales están disponibles en el sitio web de MARN (www.marn.gob.sv). Sin embargo, en la práctica la capacidad gubernamental de revisar con cuidado los pedidos para el cambio en el uso de la tierra es muy limitada y la gran mayoría son aprobados (Blackman, et.al. 30). En términos de recursos hídricos, el país depende casi totalmente del Río Lempa y gran parte de las aguas del país están contaminadas (Orellana).

Ha habido movilizaciones medioambientales ciudadanas de gran impacto durante la época de la posguerra. Dos salvadoreños han ganado el Premio Goldman: Ricardo Navarro en 1995, por su trabajo con el Centro Salvadoreño de Tecnología Apropiada (CESTA) y Francisco Pineda, en 201 1, por su liderazgo en el Comité Ambiental de Cabañas (Goldman Environmental Prize). Otras movilizaciones salvadoreñas de índole medioambiental incluyen el Movimiento de Víctimas, Afectados y Afectadas por el Cambio Climático (MOVIAC) y la "Mesa Nacional Frente a la Minería," que ha aglutinado a grupos comunitarios tradicionales, líderes católicos y estudiantiles y aliados internacionales, para lograr victorias importantes; esta última movilización será el foco de los siguientes párrafos.

La "Mesa" ha atraído la atención internacional de la prensa, en gran parte por la demanda legal que presentó una compañía minera que vio bloqueada su explotación de recursos minerales por la movilización anti-minera en El Salvador. La cobertura, en inglés, de las publicaciones NACLA (Achtenberg) y The Nation (Broad y Cavanagh), detallan la historia de la "Mesa" y la demanda legal, bajo provisiones de DR-CAFTA, de Pacific Rim Mining (y su sucesora Oceana Gold). En 2007 una encuesta nacional llevada a cabo por la Universidad Centroamericana reveló una fuerte oposición ciudadana a la minería (Achtenberg 4) y meses después, en 2008 la "Mesa" logró que el Presidente Tony Saca declarara su oposición a la minería metálica en el país. Esta oposición presidencial frente a la minería metálica ha continuado con los dos siguientes presidentes y ahora la "Mesa" busca que se apruebe un proyecto de ley para prohibir la minería 
metálica con el fin de no depender de la posición presidencial al respecto.

La misión de la "Mesa", según su sitio web, es la siguiente:

trabajar como alianza política estratégica para erradicar la minería metálica El Salvador mediante la aprobación de una ley que prohíba la exploración y explotación de minería metálica en El Salvador, las reformas [sic] de leyes pertinentes para la regulación de la minería no metálica de manera que se minimicen los impactos al medio ambiente, que amplíe y garantice la información y participación ciudadana en la toma de decisiones; el acceso a la justicia de las victimas y familiares de las victimas ocasionadas por la lucha ambiental en El Salvador; y la incidencia en los gobiernos vecinos para que se prohíba la explotación de proyectos mineros en las cuencas compartidas con El Salvador ("Mesa").

Cabe señalar que su retórica visual es sencilla y "pegajosa" en términos comunicativos: modifica la imagen del rótulo público por "Peligro" (por ejemplo, "Peligro: Alto Voltaje" o "Peligro: Productos Tóxicos"). El triángulo dorado de la "Mesa" tiene en la parte superior una calavera, los huesos en forma de " $X$ " abajo se componen en parte por las palabras "minería metálica" y debajo aparece la figura del país. La cuenta de Twitter @ no_mineria_sv está activa desde 2011 , cuenta con casi 1.700 seguidores en 2016 y sirve como herramienta de difusión de materiales en otras plataformas como Calaméo, una plataforma comercial para la difusión de publicaciones e informes digitales.

\section{Honduras}

Honduras comparte cuencas hídricas con El Salvador (y Guatemala) y se ubica en el mismo "cinturón de oro" centroamericano que sus vecinos. En términos ecológicos generales se estima que sólo el 15\% del territorio nacional es adecuado para la agricultura mecanizada; los valles más productivos están controlados por una minoría agroexportadora y pequeños terratenientes de subsistencia se encuentran en las colinas y montañas (Cherrett 223). Honduras perdió casi el $25 \%$ de sus bosques entre $1980-1990$ (Tucker 207) y ha tenido durante años la más alta tasa de desforestación en Centroamérica (Lineal y Laituri 60). En la zona occidental del país, sitio de marcados conflictos medioambientales, se estima que el $97 \%$ de la tierra es inadecuada para la agricultura intensiva, siendo más apta para bosques de pinos y robles (Tucker 207).

En términos de política ecológica, en Honduras se creó en 1974 Fo- 
restry Development Corporation (COHDEFOR) (Tucker 221) y en 1987 se prohibió la tala (Southwork y Tucker 277). Durante la presidencia de Rafael Callejas (1990-1994) hubo una respuesta institucional a la tala, bajo la forma de la plantación de árboles en las plazas, muchas veces con mensajes escritos sobre su importancia en anotaciones públicas (Bass 567). A pesar de importantes esfuerzos realizados a favor de la reforestación, mucha gente sólo tiene acceso a bosques comunales (Southwork y Tucker 276) y ha habido múltiples casos de tala ilegal, como la tala a la que se opuso el Movimiento Ambiental de Olancho (Bono), por cuyo liderazgo Fr. José Andrés Tamayo Cortés, ganó el Premio Goldman. Asimismo, nació una importante organización facilitadora de la movilización medioambiental, el Consejo Cívico de Organizaciones Populares e Indígenas de Honduras (COPINH), organización cuya labor inicia durante las luchas comunitarias contra la tala en la zona occidental de Intibucá durante los años 90 y cuyo trabajo es el foco de esta sección (Gregorcic 362).

Antiguas divisiones de orden nacional y de muy larga duración siguen teniendo un impacto enorme en cuanto al acceso al control de recursos y a una voz en su gestión. Con el aumento en el precio del oro, entre 20022012, se han acelerado en Honduras dos proyectos interrelacionados: la explotación minera de oro, con concesiones otorgadas aceleradas después del golpe de estado en 2009 y una nueva ley de minería en 2013, y la construcción actual o planeada de decenas de centrales hidroeléctricas (para proveer los proyectos mineros de electricidad de bajo costo), lo que ha movilizado redes de colaboradores en su contra durante la segunda década del Siglo XXI (COHA).

Cabe mencionar que COPINH opera en el contexto de una movilización multimodal medioambiental entre una variedad de otros grupos y redes en Honduras, incluyendo el Observatorio de los pueblos indígenas de Honduras; el Comité para la Defensa y Desarrollo de la Flora y Fauna del Golfo de Fonseca (CODDEFFAGOLF), ganador en 2015 del Premio Ecuatorial de Programas de las Naciones Unidas para el Desarrollo (PNUD); la Organización Fraternal Negra Hondureña (COFRANEH), organización de activismo garífuna y otras. A pesar de operar en un contexto de amenazas frecuentes en su contra (Human Rights Watch; Malkin y Arce), COPINH ha coordinado y colaborado con la actividad de 400 comunidades, en 38 municipios, en Honduras desde su fundación (Gregorcic 362). El territorio lenca ha sido el epicentro de la movilización multimodal en contra de los nuevos megaproyectos asociados con la minería y en particular, desde 2013, con la oposición al proyecto hidroeléctrico Agua Zarca en el Río Gualcarque. 
En adición a su trabajo organizativo y educativo al lado de múltiples comunidades en el país, COPINH ha tenido una difusión multimodal amplia: blogs en inglés y español, presencia en Twitter y Facebook; ocupación de carreteras; consistente uso de retórica visual de resistencia y de afirmación de identidades indígenas y de la representación artística de mártires, como Tomás García, asesinado por fuerzas de seguridad nacional en 2013, en murales, pancartas y banderas, y en memes digitales. Su sitio web y los blogs de COPINH sirven como repositorios y archivos de acciones, entrevistas, videos musicales, informes de noticieros y declaraciones de solidaridad de organizaciones con sede en otros países, como Alemania, los Estados Unidos, los Países Bajos; difusión de noticias en Radio Gualcarque; y participación en conferencias y encuentros internacionales. Los sitios web sirven además para facilitar la colaboración internacional horizontal alrededor de temas medioambientales y para hacer posible el aprendizaje mutuo por medio de "horizontal networks of interactive communication" (Castells Communication 7). Documentales en cortometraje como "Mother of All Rivers" (2015) y "The New Environmentalists" (2013) y música producidos con tecnología costosa por individuos y entidades fuera de la zona y disponibles gratis en plataformas como Vimeo, pueden difundirse ampliamente por redes sociales. Enlaces a documentales producidos, a vídeos de aceptación de premios como el Goldman, a entrevistas, sirven también como una forma de protección contra las amenazas y campañas de desprestigio, ya que estos artefactos, curados por organizaciones comunitarias y/o sin fines de lucro en sitios web de fácil acceso, introducen una dinámica transnacional a lo que gobiernos locales y nacionales pudieran plantear como una batalla local y contra la cual pudieran usar tradicionales fuerzas de opresión sin mucha oposición. Se observa, después del asesinato de Berta Cáceres, que las denuncias multitudinarias muchas veces incluyen la mención de las amenazas que ha habido en contra de los participantes en COPINH por muchos años, con enlaces a discursos y vídeos en los cuales Berta Cáceres y COPINH han figurado denunciando estas mismas amenazas contra sus vidas.

El discurso de aceptación del Premio Goldman de Berta Cáceres, tuiteado y cargado en actualizaciones de Facebook después de su muerte, es el ejemplo por excelencia de la difusión de una epistemología y ética alternativas para la movilización multimodal en defensa del patrimonio biocultural centroamericano. Cito del discurso de aceptación, este fragmento:

En nuestras cosmovisiones somos seres surgidos de la tierra, del agua y del maíz. De los ríos somos custodios ancestrales el pueblo Lenca, resguardados además por los espíritus de las niñas que nos enseñan que dar la vida de múltiples formas por la defensa de los ríos es dar la vida por el 
bien de la humanidad y de este planeta. El COPINH caminando con los pueblos por su emancipación ratifica el compromiso de seguir defendiendo el agua, los ríos, nuestros bienes comunes y la naturaleza. (Goldman)

Se observa en su discurso un alineamiento con epistemologías alternativas y un elemento de activismo de género, que también se encuentra en otras movilizaciones medioambientales en las Américas (Contagio Radio). Vemos con los esfuerzos de COPINH y otros grupos que las redes digitales facilitan alianzas con otras entidades, por ejemplo, la Red de Defensoras de Mesoamérica y el Comité por la Libre Expresión. Días antes del asesinato de Berta Cáceres, la Red de Defensoras de Honduras circuló el "Pronunciamiento de Movimientos Sociales y Populares de Honduras (PMSPH) ante la realidad de la minería en nuestro país" en la que declaró que "toda concesión minera se traduce en herencia de muerte" y denuncia "la proliferación voraz de la industria minera en connivencia con la secretaría del Ambiente, las municipalidades y las Fuerzas Armadas de Honduras". La denuncia circulada, además de tomar una posición contra la explotación minera, sirve como comunicado informacional, sobre derrames, expropiaciones, amenazas, igual que las victorias y acciones en relación con tales eventos.

\section{Guatemala}

Las presentes condiciones e historia medioambiental de Guatemala incluyen un alto número de nuevas licencias de minería en un contexto político de corrupción, impunidad y represión violenta de protestas comunitarias. Entre 2012-2013 el Ministerio de Energía y Minas (bajo el mandato presidencial de Otto Pérez Molina) aprobó 387 licencias para la exploración y explotación de minería metalúrgica, a pesar de la oposición de un millón de guatemaltecos que se oponían a la minería en sus comunidades (Pedersen 188). Ante este hecho se ha aumentado la resistencia a la minería y el extractivismo en general a través de movimientos en defensa de modos de vida amenazados y también territorios sagrados, fenómeno estudiado en múltiples publicaciones académicas, activistas y religiosas (Boyd; Dougherty; Glynn; Holden y Jacobson "Civil Society Opposition"; Holden y Jacobson "Ecclesial Opposition"; Pedersen; Roberts; Snell; Urkidi).

La movilización anti-minera ocurre en un contexto socio-político que ha presenciado en Guatemala el empleo de medios digitales en campañas con respecto a temas que se podrían caracterizar por su tendencia general a denunciar la corrupción y demandar una voz ciudadana en el manejo de los recursos del país. El más destacado ejemplo sería quizás la campaña en redes sociales \#RenunciaYa que logró fomentar una de las protestas más 
grandes en la historia del país (Goldman, F.). El hecho de que renunciara el Presidente Otto Pérez Molino ante la presión ciudadana movilizada en parte por medios sociales, sugiere cuáles son las posibilidades disruptivas de los medios digitales más básicos en manos de una población harta de su marginación.

Existen múltiples grupos de activismo comunitario biocultural y/o ambientalista en Guatemala, incluyendo SAVIA, donde ha tomado un papel importante Magalí Rey Rosa, hermana del conocido escritor Rodrigo Rey Rosa. SAVIA produjo un mapa de seis temas medioambientales y ha usado el mapa como herramienta de fortalecimiento y educación; posee una presencia en Facebook y sitio web. Otras organizaciones con presencia digital incluyen Defensores de la naturaleza; Guía ecológica; y el Comité de Unidad Campesina. Cabe destacar también "El movimiento pacífico de justicia medioambiental 'La Puya'", iniciado luego de que un miembro de la comunidad, Estela Reyes, aparcara su carro en la entrada a la mina El Tambor (Guatemalan Human Rights Commission). El movimiento se cobró una victoria importante en 2016 cuando la Corte Suprema de Guatemala suspendió provisionalmente la licencia de la compañía estadounidense Kappes, Cassiday \& Associates.

Las publicaciones emitidas por el Comité de Unidad Campesina (CUC) en 2008 ejemplifican la retórica medioambiental de muchas movilizaciones centroamericanas al articular una defensa del derecho a la tierra y el agua para comunidades campesinas $\mathrm{y} / \mathrm{o}$ indígenas, al denunciar la complicidad del estado con las compañías multinacionales extractivistas y al enfatizar el papel de las comunidades tradicionales como defensores de la naturaleza. Cito del "Manifiesto por la soberanía de nuestros pueblos y la defensa de la Madre Naturaleza" aquí:

Ahora hemos planteado y seguiremos planteando que nuestras comunidades y territorios se ven amenazadas con la explotación minera y petrolera, la construcción de megaproyectos y proyectos turísticos, la expansión de los monocultivos como la caña de azúcar o la palma africana y la apropiación de las riquezas naturales, que por generaciones hemos cuidado. Estas empresas están desarrollando un nuevo despojo organizado y ejecutado por terratenientes y empresarios nacionales y transnacionales con la complicidad de funcionarios gubernamentales.

Documentales como Heart of Sky, Heart of Earth (2011) y El oro o la vida (201 1), este último disponible en YouTube, captan las mismas críticas y más, en las voces de gente de las comunidades (muchas veces indígenas) afectadas por enormes transformaciones medioambientales impulsadas por las fuerzas de mercado. 
Fig. 1 Activismo mutimodal

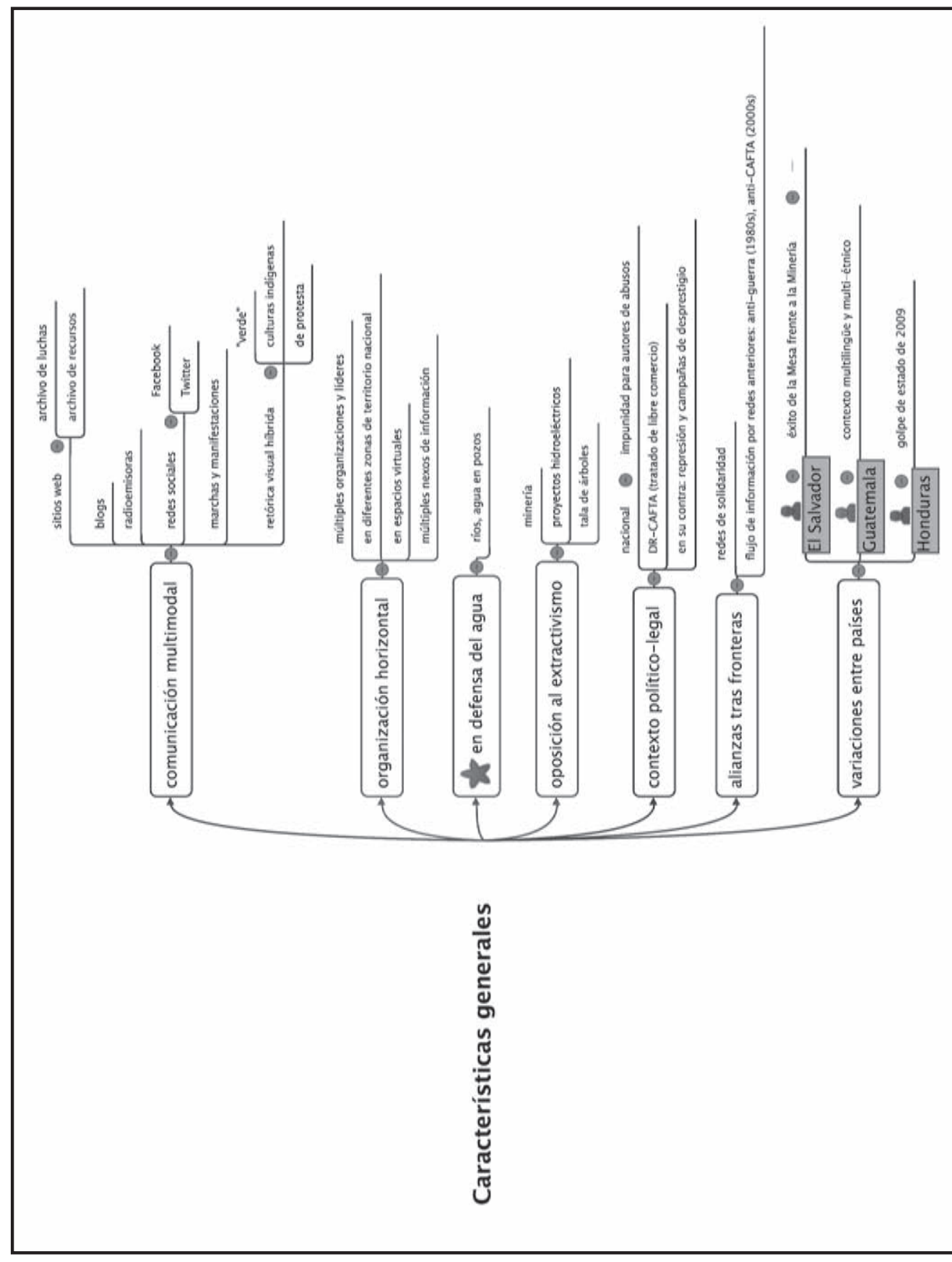




\section{Discusión: Características generales e implicaciones de la movilización MULTIMOdAL EN DEFENSA DEL PATRIMONIO BIOCULTURAL}

Hay ciertas características generales que identifican la movilización multimodal medioambiental en Centroamérica, por ejemplo, su uso de medios sociales como Twitter y Facebook con bajas barreras de entrada para usuarios, así como sitios web, para archivar información y diseminar llamadas a acción. Cumplen así una importante función social, informativa y educativa, ante la escasez de información pública disponible para la ciudadanía -El Salvador y Guatemala ocupan el sitio \#71 en el Global Open Data Index (Honduras no figura en la lista) (Open Knowledge)- y las limitaciones de los medios tradicionales (sean económicos o políticos). Asimismo, como emisoras y repositorios de información agregada por organizaciones comunitarias y sus colaboradores individuales, las redes digitales activadas por organizaciones y alianzas tradicionales, así como por formas de organización hechas posibles gracias a las masas (como campañas de hashtag) funcionan para democratizar el acceso a la información y la capacidad creativa en términos de creación de contenido.

A diferencia de las formas de organización en la época pre-digital, el potencial de la escala de difusión de la información archivada por movilizaciones multimodales es inmenso. Por otro lado, en un contexto donde, por ejemplo, no hay ni un solo corresponsal extranjero permanente en Honduras (Radio Ambulante), la presencia de voces informadas, equipadas con tecnología básica (muchas veces, un simple teléfono móvil) y arraigadas en situaciones en las que entidades poderosas tienen el interés en no difundir información, vuelve a ser una fuerza que paulatinamente va cambiando las reglas del juego para todos los actores como se ha visto en la reacción del mundo frente al asesinato de Berta Cáceres o, en otro contexto, en el éxito del movimiento \#RenunciaYa en Guatemala. También el hecho de que movimientos medioambientales en el interior de estos países tengan en el Siglo XXI la capacidad de transmitir y filtrar información rápidamente y tras fronteras, tiene enormes implicaciones en términos de posibilidades de disrupción de mecanismos tradicionales de control. Cobran aún más importancia las movilizaciones digitales cuando los defensores del medioambiente desempeñan su actividad en un contexto de extrema violencia como es el del Triángulo norte. Honduras, por ejemplo, se caracteriza por ser el país de mayor peligro para los defensores del medio ambiente (Global Witness) y ha habido una activación de redes digitales para presionar al estado hondureño a investigar a fondo el asesinato de Berta Cáceres, así como a dejar salir del país al activista medioambiental mexicano Gustavo Castro, herido en el mismo asalto (Alliance for Global Justice). 
Por lo general, se observa una destacada presencia de la denuncia política en los discursos de redes de acción medioambiental: una crítica de impunidad, de violaciones de derechos humanos y civiles, de clientilismo y corrupción, así como una insistencia en una zona con inclusión social muy baja según índices como el que publica Americas Quarterly, de señalar el nexo entre inequidad, explotación extractivista de recursos naturales y corrupción política. Los datos del 2014 revelan que El Salvador, Paraguay, Honduras y Guatemala son los últimos en la lista de inclusión social compuesta por la Americas Society (58), hecho que acarrea graves implicaciones si se considera que, según expertos, la pobreza ha sido el más importante determinante de exclusión para la gran mayoría de los centroamericanos, con impacto en su capacidad de participar en decisiones y controlar los recursos que les proveen de subsistencia (Charrett 221). Si no se rompe el ciclo de exclusión y explotación, asevera el geógrafo económico y especialista en Centroamérica lan Charrett, "environmental collapse is imminent" [el colapso medioambiental es inminente] (225).

Se puede entender, entonces, a las intervenciones multimodales a favor del patrimonio biocultural en América Central como un fenómeno cultural que le otorga poder a la sociedad civil frente a la ausencia de instituciones fuertes (en particular, jurídicas, escolares/educativas e informáticas) y que da testimonio del colapso medioambiental de zonas enteras del territorio del istmo. La práctica ética, estética y comunicativa que implica el activismo multimodal centroamericano demanda a su vez que se le preste atención al nexo entre la deshumanización y la depredación del medio ambiente. Las intervenciones, por lo general, insisten en postular no sólo una denuncia de esta dinámica desde el locus de sus efectos más nocivos, sino también de articular epistemologías y éticas alternativas a las utilitarias: las católicas/ cristianas que revindican la hermandad de los seres humanos y una responsabilidad para con el prójimo y la Tierra; las indígenas, que comprenden una dinámica en que los seres humanos tienen agencia en el mundo biofísico tanto como cada parte de ese mundo, desde los ríos a los animales. Por eso, caracterizo muchas de las intervenciones, creadas in situ (como manifestaciones o murales) o en la red (como pronunciamientos o memes) de parte de participantes y/o colaboradores en las movilizaciones estudiadas aquí, como actos de descolonización ya que le niegan supremacía a una sola visión del mundo e insisten en una co-participación de una sociedad pluralista.

Cabe resaltar también que la dimensión digital ayuda a crear un espacio alternativo donde se encuentren disidentes de la política económica en los países que sirven de sedes para compañías multinacionales y disidentes 
en las comunidades afectadas por sus inversiones, ejemplo del cual sería el activismo canadiense anti-minero y las redes transnacionales que se han formado en contra de las compañías canadienses en Centroamérica (Pedersen 192). Hay una historia de casi medio siglo de redes transnacionales de solidaridad que datan de las guerras de los 70 s y 80 s e incluyen las de la lucha contra CAFTA de los 2000 (Finley-Brook and Hoyt); lo que diferencia la actividad ahora es la facilidad en la comunicación y las múltiples plataformas digitales disponibles para la diseminación de información.

Como otros movimientos de la era de la sociedad red, desde las protestas estudiantiles en Chile a la Primavera Árabe, existe la posibilidad de levantar la voz en los nuevos medios y sentir la presencia inmediata de colaboradores, algo que Castells enfatiza es imprescindible para superar el miedo del individuo ante la posibilidad de represión (Castells, Networks 14-15). De suma importancia para muchas movilizaciones es la posibilidad que brindan los medios digitales de transformar los abusos extremos en armas contra los abusadores, ya que los actos de violenta represión, testimoniados "en vivo" cada vez que un usuario de Internet hace clic en un enlace, pueden despertar una respuesta emocional y así añadir a nuevos simpatizantes al movimiento. Ha sido el caso muchas veces que los actos de extrema violencia catalizan la oposición (solo hay que pensar en los asesinatos de Oscar Romero y Pedro Joaquín Chamorro para tener a mano dos ejemplos de América Central) pero los medios digitales cambian el escenario y facilitan la amplia difusión, casi inmediata, de los actos captados en cámaras y videocámaras en manos de ciudadanos; les da un lugar en que existir permanentemente en archivos digitales del Internet; y bajan las barreras de entrada para la participación solidaria de simpatizantes.

\section{CONCLUSIÓn}

Las sociedades no son comunidades con intereses y valores compartidos, observa Castells, sino que son estructuras que toman forma de los conflictos y negociaciones entre diversos actores; nunca terminan los conflictos, solamente pausan en acuerdos temporarios que los actores más poderosos intentan transformar en instituciones para lograr sus fines de dominio (Communication 14). El espacio digital del Internet, con sus varias plataformas y aplicaciones, permite a grupos subordinados tener una plataforma para insistir en mostrar pluralidades invisibilizadas por las instituciones sociales y cobrar importancia como agentes en la sociedad red. La sociedad red, Castells insiste, es maleable pero lo que persiste es su dominio sobre las actividades y las personas que son externas, a menos que estas personas, de comunidades locales, se conectan al fenómeno global por medio de redes 
globales alternativas elaboradas por movimientos sociales (Communication 26). La movilización popular en defensa del patrimonio biocultural, en que han sido pioneros grupos y redes populares como los que estudio aquí, y muchos otros en otras partes del mundo, representa un esfuerzo importante en la construcción de redes alternativas, a favor de la sustentabilidad de la población humana en toda su diversidad en el planeta.

El estudio de movimientos multimodales en defensa del patrimonio biocultural presenta la posibilidad de enriquecer las humanidades y las ciencias sociales medioambientales. Son meritorios de consideración a fondo no sólo los movimientos que menciono aquí de manera preliminar, sino también otros recursos digitales generales como el sitio curado BiodiversidadLA; el Observatorio Latinoamericano de Conflictos Medioambientales; Radio Contagio (sitio web y señal); y el Movimiento Mesoamericano contra el Modelo Extractivo; y sus redes de colaboradores dentro y fuera de la región. En cuanto al emergente campo de las humanidades digitales en la región (Galina Russell), será imprescindible que sus proyectos de mapeo, de digitalización y curación de materiales y de creación artística se realicen en colaboración con grupos locales y que se extiendan a espacios populares de capacitación, como el de los centros comunitarios. Asimismo, el trabajo de salvaguardar el patrimonio biocultural en diversos rincones del planeta, en términos tanto culturales como biológicos, y de anclar este trabajo de conservación en la descolonización del conocimiento es una acción a favor de una sociedad red inclusiva y animada, activa y activada para encarar y enfrentar los debates importantes sobre los cambios medioambientales del presente siglo.

\section{Bibliografía}

ACHTENBERG, Emily (201 1), "A Mining Ban in El Salvador?" NACLA Report on the Americas 44, 5, pp. 3-4.

Alliance for Global Justice (2016): "Berta Cáceres: Indigenous Leader One More Victim of US-Approved Coup in Honduras." Accessed 2016 March 6. http://afgi.org/berta-caceres-indigenous-leader-one-more-victim-of-us-approved-coup-in-honduras.

BARBAS-RHODEN, Laura (2011), Ecological Imaginations in Latin American Fiction. University Press of Florida, 195 pp.

BASS, Joby (2005), "Message in the Plaza: Landscape, Landscaping, and Forest Discourse in Honduras." Geographical Review 95, 4, pp. 556-77. BENITTEZ, José Luis (2012), "Salvadoran Transnational Families: ICT and Communication Practices in the Network Society." Journal of Ethnic and Migration Studies 38, 9, pp. 14-39. 
BENNETT, Professor W. Lance, and Dr Alexandra Segerberg (2013), The Logic of Connective Action: Digital Media and the Personalization of Contentious Politics. Cambridge University Press, 240 pp.

BLACK, Eric, and Frauke Sandig (2011), Heart of Sky, Heart of Earth. Documentary.

BLACKMAN, Allen, Beatriz Ávalos-Sartorio, and Jeffrey Chow (2007), "Shade Coffee \& Tree Cover Loss: Lessons from El Salvador." Environment 49, 7, pp. 22-33.

BOYD, Stephanie (2012), "And the Birds Dropped Dead from the Sky." New Internationalist, 456, pp. 38-40.

BROAD, Robin, and John Cavanagh (2014), "A Strategic Fight Against Corporate Rule." Nation 298, 5, pp. 21-25.

BRONDO, Keri (2013), "Land Loss and Garifuna Women's Activism on Honduras' North Coast." Journal of International Women's Studies 9, 1, pp. 99-116.

CARACOL PRODUCCIONES (2012), El Oro O La Vida / Life for Gold (Completo) Spanish with English Subtitles. https://www.youtube.com/wat$c h ? v=C A D S+D t d p 7 c$.

CASTELLS, Manuel (2009), Communication Power. Oxford: Oxford University Press, $574 \mathrm{pp}$.

CASTELLS, Manuel, (2012), Networks of Outrage and Hope: Social Movements in the Internet Age. Polity, 300 pp.

CHERRETT, lan, (2001), "Decentralization, Rural Poverty, and Degradation of Uplands in Central America." Mountain Research and Development 21, 3, pp. 221-25.

COHA (2015): "The Dangerous Path Toward Mining Law Reform in Honduras." COHA. December 18. Accessed 16 March 2016. http://www.coha. org/the-dangerous-path-toward-mining-law-reform-in-honduras/.

CONTAGIO RADIO (2016): "Indígenas Hondureños Defienden El Río Gualcarque de Construcción de Represa." Accessed 2016 February 11. http:// www.contagioradio.com/indigenas-hondurenos-defienden-el-rio-gualcarque-de-construccion-de-represa-articulo-16080/.

DIARIO LA PRENSA (2016): "'La Mataron Por Su Lucha': Madre de Berta Cáceres." Accessed 2016 March 14. http://www.laprensa.hn/sucesos/935886-410/la-mataron-por-su-lucha-madre-de-berta-cáceres.

DOGGETT, Martha (201 1): "Contending with Conflict." Harvard International Review 33, 3, pp. 72-72. 
DOUGHERTY, Michael L. (2011), "The Global Gold Mining Industry, Junior Firms, and Civil Society Resistance in Guatemala." Bulletin of Latin American Research 30, 4, pp. 403-18.

FINLEY-BROOK, Mary, and Katherine HOYT (2009), "CAFTA Opposition: Divergent Networks, Uneasy Solidarities." Latin American Perspectives 36, 6, pp. 27-45.

GLYNN, Tracy (2013), "It's Bigger than Bling Bling and the Banks." Women \& Environments International Magazine, 92/93, pp. 23-25.

GLOBAL WITNESS (2016): "How Many More?" Accessed 2016 March 14. https://www.globalwitness.org/en/campaigns/environmental-activists/how-many-more/.

GOLDMAN ENVIRONMENTAL FOUNDATION (2016) "Prize Recipients." Accessed March 14. http://www.goldmanprize.org/prize-recipients/. GOLDMAN ENVIRONMENTAL PRIZE (2014), Berta Caceres Acceptance Speech, 2015 Goldman Prize Ceremony. https://www.youtube.com/watch? $\mathrm{v}=A R 1 \mathrm{kw} \times 8 \mathrm{~b} 0 \mathrm{~ms} \& f$ feature=youtu.be.

GOLDMAN, Francisco (2015), "From President to Prison: Otto Pérez Molina and a Day for Hope in Guatemala." The New Yorker, September 4. http://www.newyorker.com/news/news-desk/from-president-to-prison-otto-perez-molina-and-a-day-for-hope-in-guatemala.

GREGORCIC, Marta (2009), "Cultural Capital and Innovative Pedagogy: A Case Study among Indigenous Communities in Mexico and Honduras." Innovations in Education \& Teaching International 46, 4, pp. 357-66.

GUATEMALA HUMAN RIGHTS COMMISSION (2016): "'La Puya' Environmental Movement". Accessed 2016 March 14. http://www.ghrc-usa.org/ our-work/current-cases/lapuya/.

HEFFES, Gisela (2014), "Introducción: para una ecocrítica latinoamericana." Revista de crítica literaria latinoamericana 79, pp. 1 1-34.

HOLDEN, William N., and R. Daniel JACOBSON (2009), "Ecclesial Opposition to Nonferrous Mining in Guatemala: Neoliberalism Meets the Church of the Poor in a Shattered Society." Canadian Geographer 53, 2, pp. 145 64.

HOLDEN, William, and R. Jacobson (2008), "Civil Society Opposition to Nonferrous Metals Mining in Guatemala." Voluntas: International Journal of Voluntary \& Nonprofit Organizations 19, 4, pp. 325-50.

HUMAN RIGHTS WATCH (2016): "Honduras: Investigate Environmental Activist's Killing." Human Rights Watch. Accessed 2016 March 10. https://www.hrw.org/news/2016/03/04/honduras-investigate-environmental-activists-killing. 
LINEAL, Matthew, and Melinda LAITURI (2013), "Community-Based Integrated Fire Management Planning: Linking Ecology and Society in Honduras." Community Development Journal 48, 1, pp. 58-74.

MALKIN, Elisabeth, and Alberto ARCE (2016): "Berta Cáceres, Indigenous Activist, Is Killed in Honduras." The New York Times, March 3. http://www. nytimes.com/2016/03/04/world/americas/berta-caceres-indigenous-activist-is-killed-in-honduras.html.

"Manifiesto Del CUC a 30 Años de Luchas" - ManifiestoCUC30aniversario. pdf." Accessed 2016 March 8. http://www.cuc.org.gt/documentos/ManifiestoCUC30aniversario.pdf.

"Mining Watch Calls on Canada Pension Plan to Divest from Tahoe Resources | Rights Action." Accessed 2016 March 11. http://www.rightsaction. org/action-content/mining-watch-calls-canada-pension-plan-divest-tahoe-resources.

"Mother of All Rivers". (2015) Mill Valley Film Group.

"The New Environmentalists". (2103) Mill Valley Film Group.

OPEN KNOWLEDGE (2016): "Open Data Index - Open Knowledge." Open Data Index. Accessed March 11. http://index.okfn.org/.

ORELLANA, Claudia (2006):"Central American Dam Project Relaunched." Frontiers in Ecology and the Environment 4, 5, pp. 228-228.

PEDERSEN, Alexandra (2014): "Landscapes of Resistance: Community Opposition to Canadian Mining Operations in Guatemala." Journal of Latin American Geography 13, 1, pp. 187-214.

RADIO AMBULANTE (2015): "The Correspondent." Radio Ambulante. Accessed 2016 March 11. http://radioambulante.org/en/audio-en/the-correspondent.

RALEY, Rita (2009): Tactical Media.. Minneapolis: University of Minnesota Press, 208 pp.

ROBERTS, Tom (2013): "A Church That Requires a Different Kind of Bishop." National Catholic Reporter 49, 24, pp. 5-6.

RUSSELL, Isabel Galina (2015): "Creating a Regional DH Community A Case Study of the RedHD" 9, 3. http://digitalhumanities.org/dhq/ $\mathrm{vol} / 9 / 3 / 000221 / 000221 . \mathrm{html}$.

SNELL, Marilyn Berlin (2007): "Bulldozers \& Blasphemy." Sierra 92, 5, pp. 36-76. SOUTHWORTH, Jane, and Catherine TUCKER (2001): "The Influence of Accessibility, Local Institutions, and Socioeconomic Factors on Forest Cover Change in the Mountains of Western Honduras." Mountain Research and Development 21, 3, pp. 276-83. 
TUCKER, Catherine M. (1999): "Private versus Common Property Forests: Forest Conditions and Tenure in a Honduran Community." Human Ecology 27, 2, pp. 201-30.

URKIDI, Leire (2011): "The Defence of Community in the Anti-Mining Movement of Guatemala." Journal of Agrarian Change 1 1, 4, pp. 556-80.

WORLD BANK: "Intentional Homicides (per 100,000 People) | Data | Table."Accessed 2016 March 14. http://data.worldbank.org/indicator/ VC.IHR.PSRC.P5.

WALLERSTEIN, Immanuel Maurice (1974): The Modern World-System. Academic Press. 